\title{
472139 - DEEP EXTUBATION AND INSERTION OF LARYNGEAL MASK AIRWAY REDUCES COUGHING AT EMERGENCE
}

\author{
Geneviève Brouillette, MD, Pierre Drolet, MD, FRCPC, François Donati, PhD, MD, \\ FRCPC \\ Département d'anesthésiologie, Hôpital Maisonneuve-Rosemont et Université de \\ Montréal, Montréal, QC, Canada
}

Introduction: Respiratory complications, particularly cough, are frequent when an endotracheal tube (ETT) is removed(1). Studies demonstrate that fewer complications are present when the laryngeal mask airway (LMA) is used (2). However, the LMA is not appropriate for all surgeries. Our study was designed to determine if the incidence of cough at extubation would be reduced by replacing the ETT by a ProSeal LMA before the end of the surgery.

Methods: After ethics committee approval and written consent by the subjects, we have recruited 80 adults ASA I to III, aged between 18 and 75 years requiring surgery under general anesthesia with tracheal intubation. Anesthetic drugs included propofol, fentanyl, rocuronium and sevoflurane. The patients were randomly divided into two groups. In the first group, the ETT was removed and replaced by an LMA (LMA group) 20-30 min before the end of surgery. In the second, the ETT was left in place (ETT group). Then, neuromuscular block was reversed and sevoflurane was discontinued. Adverse effects at replacement of ETT (patient movement, ventilatory difficulties, desaturation, cough, difficulty of insertion, number of trials) were recorded. At emergence, the number of cough episodes was noted. Patients were asked about hoarseness and sore throat in the recovery room and the day after the surgery.

Results: In each group, 39 patients completed the study. Demographic data were similar in both groups. Replacement of ETT by LMA was successful in all patients, only minor complications (movement) were seen in two subjects. From the cessation of sevoflurane to 5 min after removal of the airway device, 37 patients with ETT, but only 7 with LMA had cough $(\mathrm{P}<0.001)$ (Figure 1$)$. In the ETT group, 8 patients had sore throat in the recovery room and 11 the day after, compared to 3 and 5 in the LMA group (NS). However, more patients in the ETT group complained of hoarseness (29 in the recovery room, 21 the day after) than in the LMA group ( 7 and 4 respectively $(\mathrm{P}<0.001)$.

Discussion: Replacement of ETT by a LMA before emergence can be performed without major complications and greatly reduces the incidence of coughing during emergence. Moreover, a reduction of hoarseness is associated with this technique.

References: (1) Br J Anaesth.1998;80:767-775. (2)Anaesthesia 1998; 53: 540-4. 


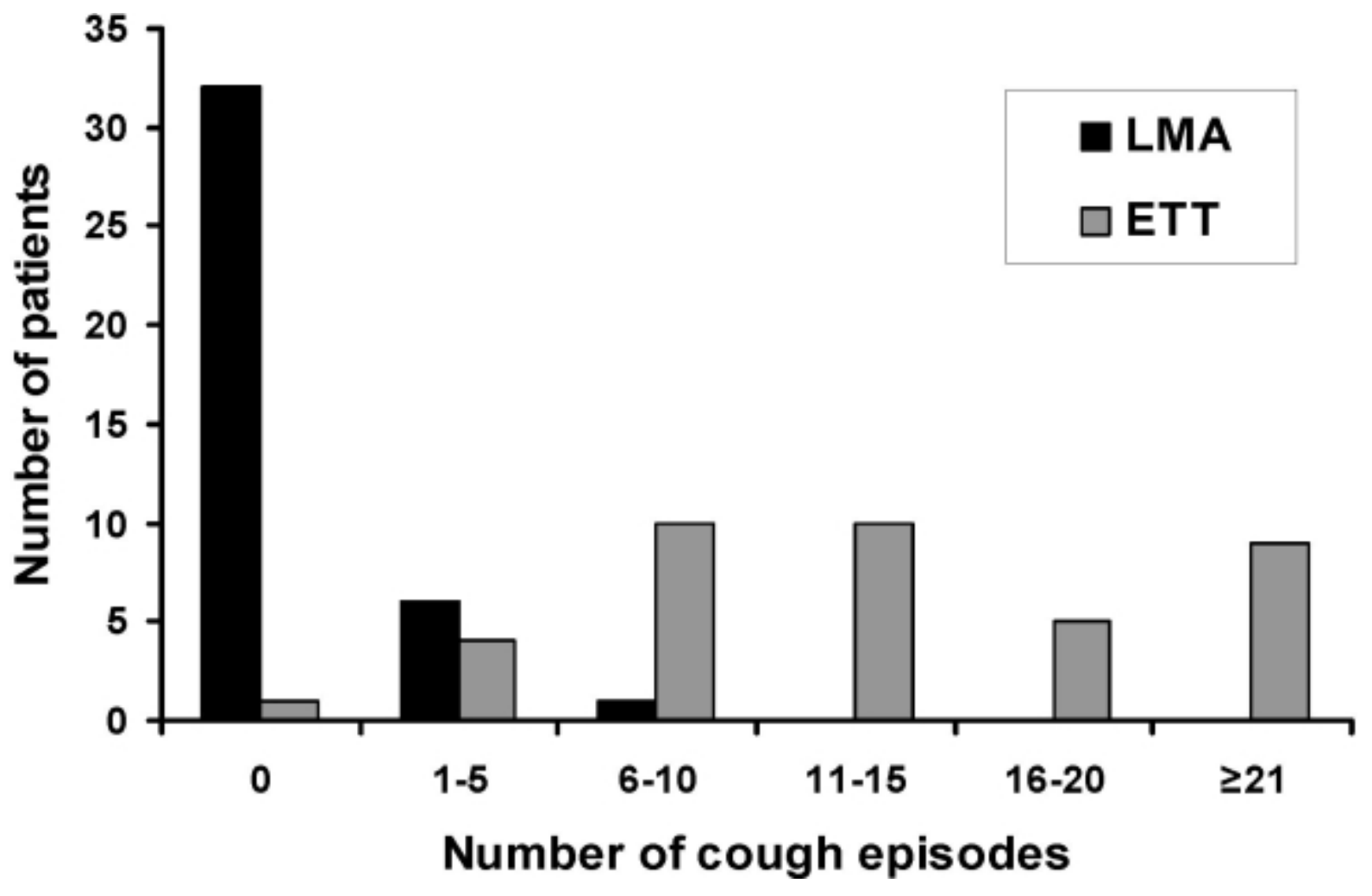

Figure 1. Number of patients versus number of cough episodes for each group. LMA : replacement of endotracheal tube by a ProSeal laryngeal mask airway; ETT : endotracheal tube left in place. $\mathrm{P}<0.001$ between the two groups. 